\title{
Modifications to the Phospholipid Composition of Saccharomyces cerevisiae Induced by Exogenous Ethanolamine
}

\author{
By S. J. RATCLIfFE, J. A. HOSSACK, G. E. WHEELER AND A. H. ROSE \\ Microbiology Laboratories, School of Biological Sciences, \\ Bath University, Bath
}

(Received 3 January 1973)

INTRODUCTION

The lipid composition of micro-organisms is very responsive to changes in the chemical and physical properties of the environment. In many micro-organisms, altering one environmental factor frequently causes multiple changes in lipid composition. For example, although lowering the growth temperature of micro-organisms below the optimum usually leads to an increased synthesis of lipids with a high proportion of unsaturated fatty acid residues (Farrell \& Rose, $1967 a, b$ ), in Saccharomyces cerevisiae this effect is accompanied by changes in the proportions of phospholipids (Hunter \& Rose, 1972). Studies in this laboratory have exploited environmentally induced changes in the lipid composition of Sacch.cerevisiae (reviewed by Hunter \& Rose, 197I) to investigate relationships between composition and function in yeast membranes. In these studies, it is desirable to be able to bring about specific changes in the lipid composition of organisms. Lester and his colleagues (Waechter, Steiner \& Lester, I969; Waechter \& Lester, I97I) recently showed that the presence of choline (I or Io $\mathrm{mM}$ ) in the growth medium leads to synthesis of an increased proportion of phosphatidylcholine by Sacch. cerevisiae. This was the first report showing how the content of one type of phospholipid in Sacch. cerevisiae can be altered by medium supplementation, and it provides a useful technique for varying the phosphatidylcholine content of yeast membranes. The present paper shows that provision of exogenous ethanolamine in the growth medium can similarly induce an increased synthesis of phosphatidylethanolamine by S. cerevisiae.

\section{METHODS}

The yeast used was Saccharomyces cerevisiae NCYC 366. Methods for maintaining the yeast, growing it in defined medium in batch culture, harvesting organisms, extracting lipids and separating and determining the contents of individual phospholipids were as described by Hunter \& Rose (1972) except that, in some experiments, the phosphorus contents of phospholipids were directly determined by acid digestion of the materials without their removal from the thin-layer plates. Phospholipids on thin-layer plates were identified from $\mathrm{R}_{p}$ values of standard compounds. Ethanolamine was sterilized by membrane filtration and included in medium at ro mM. Choline, which was included in medium at I mM, was sterilized by filtration or by autoclaving.

\section{RESULTS}

Addition of choline to the medium causes an increase of about $60 \%$ in the synthesis of total phospholipid by Saccharomyces cerevisiae NCYC 366, as indicated by the phosphorus content of the lipids (Table I). Most of this increase is attributable to an almost fourfold 
Table I. Effect of exogenous choline and ethanolamine on the phospholipid composition of Saccharomyces cerevisiae NCYC 366 and incorporation of exogeneous ethanolamine into these phospholipids Choline was incorporated into the basal medium at I mM and ethanolamine at $10 \mathrm{~mm}$. Organisms
were harvested from cultures in the mid-exponential phase of growth $(0 \cdot 20$ to $0.24 \mathrm{mg}$ dry wt $/ \mathrm{ml}$ ),
lipids extracted, and determinations made of the lipid phosphorus and the contents of individual
phospholipids. Values quoted are the means of at least four separate determinations, where
indicated \pm standard errors of the mean. In experiments on incorporation of radioactive ethanol-
amine, organisms were grown in medium supplemented with $[2-14 \mathrm{Clethanolamine} \mathrm{(} \mu \mathrm{Ci} / \mathrm{mmol})$
with or without choline. Bands of silica gel containing labelled phospholipid were scraped off thin-
layer plates, and transferred to scintillation vials containing $5 \mathrm{ml}$ scintillation liquid (toluene:
2,5 -diphenyloxazole; I:0.003, v/w). Samples were counted in a Beckman liquid scintillation
spectrometer (model 1650 ) for up to 100 min. Readings were corrected for average background count
using blank areas of silica gel from thin-layer plates.

Content ( $\mu \mathrm{mol}$ phosphorus/g dry wt yeast) in lipids from organisms grown in:

\begin{tabular}{|c|c|c|c|}
\hline $\begin{array}{l}\text { Unsupple- } \\
\text { mented } \\
\text { medium }\end{array}$ & $\begin{array}{c}\text { Medium } \\
\text { supple- } \\
\text { mented } \\
\text { with choline }\end{array}$ & $\begin{array}{l}\text { Medium } \\
\text { supple- } \\
\text { mented } \\
\text { with } \\
\text { ethanol- } \\
\text { amine }\end{array}$ & $\begin{array}{c}\text { Medium } \\
\text { supple- } \\
\text { mented } \\
\text { with } \\
\text { choline + } \\
\text { ethanolamine }\end{array}$ \\
\hline
\end{tabular}

Phospholipid

Phosphatidylcholine

Dimethylphosphatidylethanolamine

Phosphatidylethanolamine

Phosphatidylinositol

Phosphatidylserine

Cardiolipin

Phosphatidic acid

Lipid phosphorus

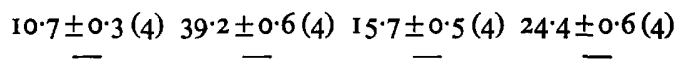

Radioactivity (c.p.m. $/ \mu \mathrm{mol}$ phosphorus) in lipids from organisms grown in:

$\begin{array}{cc}\begin{array}{c}\text { Medium } \\ \text { supple- } \\ \text { mented } \\ \text { with } \\ \text { ethanol- } \\ \text { amine }\end{array} & \begin{array}{c}\text { Medium } \\ \text { supple- } \\ \text { mented } \\ \text { with } \\ \text { choline }+ \\ \text { I339 } \\ 2336\end{array} \\ \begin{array}{c}\text { ethanolamine } \\ 558\end{array} & 0 \\ & 284 \\ 98 & 32 \\ 0 & 0 \\ 0 & 0\end{array}$

rise in the content of phosphatidylcholine. The increases in total phospholipid and in the content of phosphatidylcholine are somewhat greater than those reported by Waechter \& Lester (197I). Supplementing the medium with ethanolamine causes a smaller increase in the synthesis of total phospholipids (Table I). In these organisms, the increase is mainly attributable to synthesis of additional phosphatidylethanolamine and to a lesser extent of phosphatidylcholine. Organisms grown in medium supplemented with both choline and ethanolamine also synthesize more total phospholipid, but to a smaller extent than those grown in medium supplemented with only ethanolamine (Table I). In organisms grown in the doubly supplemented medium, the increase in phospholipid is caused mainly by an increase in the phosphatidylcholine content; synthesis of other phospholipids was hardly affected as compared with organisms grown in unsupplemented medium. Small amounts of other phospholipids, including dimethylphosphatidylethanolamine, were detected in lipids from organisms grown in the various media, but the amount of any one of these lipids never exceeded I $\%$ of the total phospholipid. Addition of choline to medium had no effect on the duration of the lag phase or on the rate of growth of the yeast. The lag phase of growth was however extended by 2 to $4 \mathrm{~h}$ when ethanolamine was added to the medium; the rate of exponential growth was not affected.

Label from $\left[2{ }^{14} \mathrm{C}\right]$ ethanolamine is incorporated into phosphatidylethanolamine by organisms grown in choline-free medium (Table I). Essentially all of the label was shown 
to be in the ethanolamine moiety of the phosphatidylethanolamine. Phospholipids were separated from neutral lipids in the extract by thin-layer chromatography (petroleum spirit + diethylether + acetic acid; 70:30:2, by vol) and hydrolysed by the procedure of Dittmer, Feminella \& Hanahan 1958. Liberated ethanolamine was separated by descending paper chromatography using methylethyl ketone + methoxyethanol $+20 \%(\mathrm{v} / \mathrm{v})$ acetic acid (40:15:20, by vol), and the spot corresponding to ethanolamine cut out and extracted with $50 \%(\mathrm{v} / \mathrm{v})$ ethanol (Magee, Baker \& Thompson, I960). The radioactivity of the ethanolamine accounted for virtually all of the activity of the phosphatidylethanolamine. The recovery did not differ significantly from that obtained when a standard of $\left[2-{ }^{14} \mathrm{C}\right]$ ethanolamine was submitted to the same procedure. However, in lipids from organisms grown in $\left[2-{ }^{14} \mathrm{C}\right]$ ethanolamine-supplemented, choline-free medium, the specific activity of phosphatidylcholine is greater than that of phosphatidylethanolamine; moreover, the specific activity of dimethylphosphatidylethanolamine is greater than that of phosphatidylcholine (Table I). In these experiments, it was not possible to resolve completely phosphatidylinositol and phosphatidylserine, and the radioactivity of these lipids is quoted as one value. When choline is added to $\left[2-{ }^{14} \mathrm{C}\right]$ ethanolamine-supplemented medium, considerably less label from ethanolamine is incorporated into the lipids (Table I), and almost all of that is in phosphatidylethanolamine the specific activity of which is nevertheless only half of that obtained with this phospholipid in extracts from organisms grown in choline-free medium.

\section{DISCUSSION}

Our data confirm the finding of Waechter et al. (1969) on the effect of exogenous choline on synthesis of phosphatidylcholine by Saccharomyces cerevisiae, and extend this effect to show that provision of exogenous ethanolamine causes a smaller but nevertheless reasonably specific increase in phosphatidylethanolamine synthesis. Organisms enriched in phosphatidylcholine or phosphatidylethanolamine will be useful in studies on the role of these phospholipids in yeast membranes, particularly since suitable methods have not so far been available for studying the role of these phospholipids in, for example, membrane transport. In addition, our data show that it is possible to label phosphatidylethanolamine in lipids of Saccharomyces cerevisiae with some degree of specificity, a technique which furnishes a valuable tool in studies on phospholipid metabolism in yeast.

Two different pathways lead to synthesis of phosphatidylcholine. One involves formation of phosphatidylserine from CDP-diacylglycerol and serine; phosphatidylserine is then decarboxylated to yield phosphatidylethanolamine which, in turn, is $N$-methylated to produce phosphatidylcholine. In a second pathway, CDP-choline reacts with a I,2-diacylglycerol to produce phosphatidylcholine; phosphatidylethanolamine is synthesized in a similar reaction involving CDP-ethanolamine and a I,2-diacylglycerol. The former or methylation pathway operates in a wide range of organisms including bacteria and fungi (Goldfine, 1972) and mammalian tissues (Lennarz, 1970). Saccharomyces cerevisiae uses the methylation pathway (Letters, 1966; Waechter et al. 1969; Hunter \& Rose, 197I). However CDP-choline and CDP-ethanolamine were detected in Sacch. cerevisiae (Kennedy \& Weiss, 1956; Tochikura, Kimura, Kawai \& Gotan, 1972) which suggested that the second or cytidine nucleotide pathway may also be used by yeast. The data of Waechter et al. (I969) and Waechter \& Lester (1971) on incorporation of choline into phosphatidylcholine provided the first direct evidence for operation of the cytidine nucleotide pathway in Sacch. cerevisiae. Our data on incorporation of label from $\left[2-{ }^{-14} \mathrm{C}\right]$ ethanolamine into phosphatidylethanolamine by Sacch. cerevisiae provide further direct evidence for operation of this pathway in this organism. Indirect evidence for synthesis of phosphatidylethanolamine from ethanolamine 
came recently from Steiner \& Lester (1972). They studied incorporation of $s n\left[{ }^{14} \mathrm{C}\right]$ glycero$3\left[{ }^{32} \mathrm{P}\right]$ phosphate into phospholipids by a cell-free particulate fraction of Sacch.cerevisiae, and calculated that the cytidine nucleotide pathway may be responsible for about a quarter of the phosphatidylethanolamine synthesized by yeast. The possibility that ethanolamine is incorporated into phosphatidylethanolamine by reactions other than those of the cytidine nucleotide pathway appears unlikely. A $\mathrm{Ca}^{2+}$-stimulated exchange of ethanolamine with phospholipids has been reported in vitro in animal (Dils \& Hübscher, 1959) and plant tissue (Yang, Freer \& Benson, 1967) although the role of the reaction in vivo is very uncertain (Hill \& Lands, 1970). The reaction has not, however, been reported in micro-organisms. Even if such an exchange reaction were possible in yeast, the rapid rate of synthesis of phospholipids in exponentially growing cultures of yeast would probably preclude any major contribution to phospholipid synthesis being made by the reaction. Nevertheless, until data are available on the operation of the exchange reaction in yeast, the possibility that some ethanolamine is incorporated in this way cannot be dismissed. The possibility that ethanolamine is incorporated into phosphatidylserine following carboxylation of ethanolamine can be discounted since an ethanolamine carboxylase has not been reported.

The increased synthesis of phosphatidylethanolamine, but to a much more limited extent of phosphatidylcholine, in organisms grown in the presence of ethanolamine suggests that methylation of phosphatidylethanolamine is under tight metabolic control. However, the high specific activity of phosphatidylcholine and especially of dimethylphosphatidylethanolamine from organisms grown in choline-free ethanolamine-supplemented medium suggests a preferential methylation of phosphatidylethanolamine synthesized from exogenously provided ethanolamine. An alternative explanation by which free ethanolamine may be methylated to choline (Nemer \& Elwyn, 1960) seems unlikely in view of the high specific activity of the dimethylphosphatidylethanolamine in these organisms. It is conceivable that methylation of phosphatidylethanolamine synthesized from phosphatidylserine, and of phosphatidylethanolamine synthesized from ethanolamine, occurs at different sites in yeast. The need for separate locations for these syntheses may be associated with the requirements of the organism for phospholipids with fatty-acid residues containing different degrees of unsaturation for, in animal tissues, it has been reported that phosphatidylcholine synthesized by the cytidine nucleotide pathway contains a greater proportion of saturated fatty-acid residues than when synthesized by the methylation pathway (Kanoh, 1969).

Our results on incorporation of labelled ethanolamine into phospholipids by organisms grown in the presence of choline support the suggestion of Waechter \& Lester (I97I) that the presence of free choline in the medium represses synthesis of enzymes that catalyse methylation of phosphatidylethanolamine to phosphatidylcholine. The contents of phosphatidylethanolamine and phosphatidylcholine in these organisms suggest that synthesis of phosphatidylethanolamine is controlled by choline since, even when methylation of phosphatidylethanolamine is repressed, the content of phosphatidylethanolamine in the organisms does not increase as compared with those grown in unsupplemented basal medium. The lower specific activity of phosphatidylethanolamine in these organisms also suggests that the presence of choline regulates synthesis of phosphatidylethanolamine from ethanolamine, possibly as a result of competition between choline and ethanolamine for a phosphokinase (Wittenberg \& Kornberg, 1953).

The work reported in this paper was supported by S.R.C. Research Grant B/RG/I82. We are grateful to Mrs Diane Arkell for competent technical assistance. 


\section{REFERENCES}

Dils, R. R. \& HüBscher, G. (1959). The incorporation in vitro of $\left[\mathrm{Me}^{-14} \mathrm{C}\right]$ choline into the phospholipids of rat-liver mitochondria. Biochimica et biophysica acta 32, 293-294.

Dittmer, J. C., Feminella, J. L. \& Hannahan, D. J. (1958). A study of the quantitative estimation of ethanolamine and serine in phospholipids. Journal of Biological Chemistry 233, 862-867.

Farrell, J. \& Rose, A. H. (I967a). Temperature effects on micro-organisms. In Thermobiology, pp. I 47-2 I 8. Edited by A. H. Rose. New York: Academic Press.

Farrell, J. \& Rose, A. H. (1967b). Temperature effects on micro-organisms. Annual Review of Microbiology 2I, IOI-I 20.

Goldfine, H. (1972). Comparative aspects of bacterial lipids. Advances in Microbial Physiology 8, I-58.

Hill, E. E. \& Lands, W. E. M. (1970). Phospholipid metabolism. In Lipid Metabolism, pp. 185-277. Edited by S. J. Wakil. New York: Academic Press.

Hunter, K. \& Rose, A. H. (1971). Yeast lipids and membranes. In The Yeasts, vol. 2, pp. 211-270. Edited by A. H. Rose and J. S. Harrison. London: Academic Press.

Hunter, K. \& Rose, A. H. (I972). Lipid composition of Saccharomyces cerevisiae as influenced by growth temperature. Biochimica et biophysica acta 260, 639-653.

KANOH, H. (1969). Biosynthesis of molecular species of phosphatidyl choline and phosphatidyl ethanolamine from radioactive precursors. Biochimica et biophysica acta $176,756-763$.

Kennedy, E. P. \& Weiss, S. B. (1956). The function of cytidine coenzymes in the biosynthesis of phospholipids. Journal of Biological Chemistry 222, I93-2 I4.

Lennarz, W. J. (1970). Lipid metabolism. Annual Review of Biochemistry 39, 359-388.

LetTers, R. (1966). Phospholipids of yeast. II. Extraction, isolation and characterization of yeast phospholipids. Biochimica et biophysica acta 116, 489-499.

MAGEe, W. L., BAKer, R. W. R. \& Thompson, R. H. S. (1960). The identification and quantitative estimation of ethanolamine and serine in lipid hydrolysates. Biochimica et biophysica acta 40, I $18-123$.

Nemer, M. J. \& ELWYN, D. (1960). The conversion of serine to ethanolamine and its derivatives in the rat. Journal of Biological Chemistry 235, 2070-2074.

Steiner, M. R. \& Lester, R. L. (1972). In vitro studies of phospholipid biosynthesis in Saccharomyces cerevisiae. Biochimica et biophysica acta 260, 222-243.

Tochikura, T., Kimura, A., Kawai, H. \& Gotan, T. (1972). Fermentative production of lipid-related substances. II. Effect of various factors on the production of cytidine diphosphate ethanolamine by yeasts. Japanese Journal of Fermentation Technology 50, 178-181.

WAEChTER, C. J. \& Lester, R. L. (I97I). Regulation of phosphatidylcholine biosynthesis in Saccharomyces cerevisiae. Journal of Bacteriology ro5, 837-843.

WAechter, C. J., Steiner, M. R. \& Lester, R. L. (1969). Regulation of phosphatidylcholine biosynthesis by the methylation pathway in Saccharomyces cerevisiae. Journal of Biological Chemistry 244, 3419-3422.

Wittenberg, J. \& Kornberg, G. A. (I953). Choline phosphokinase. Journal of Biological Chemistry 202, $431-444$.

YANG, S. F., Freer, S. \& Benson, A. A. (1967). Transphosphatidylation by phospholipase D. Journal of Biological Chemistry 242, 477-484. 